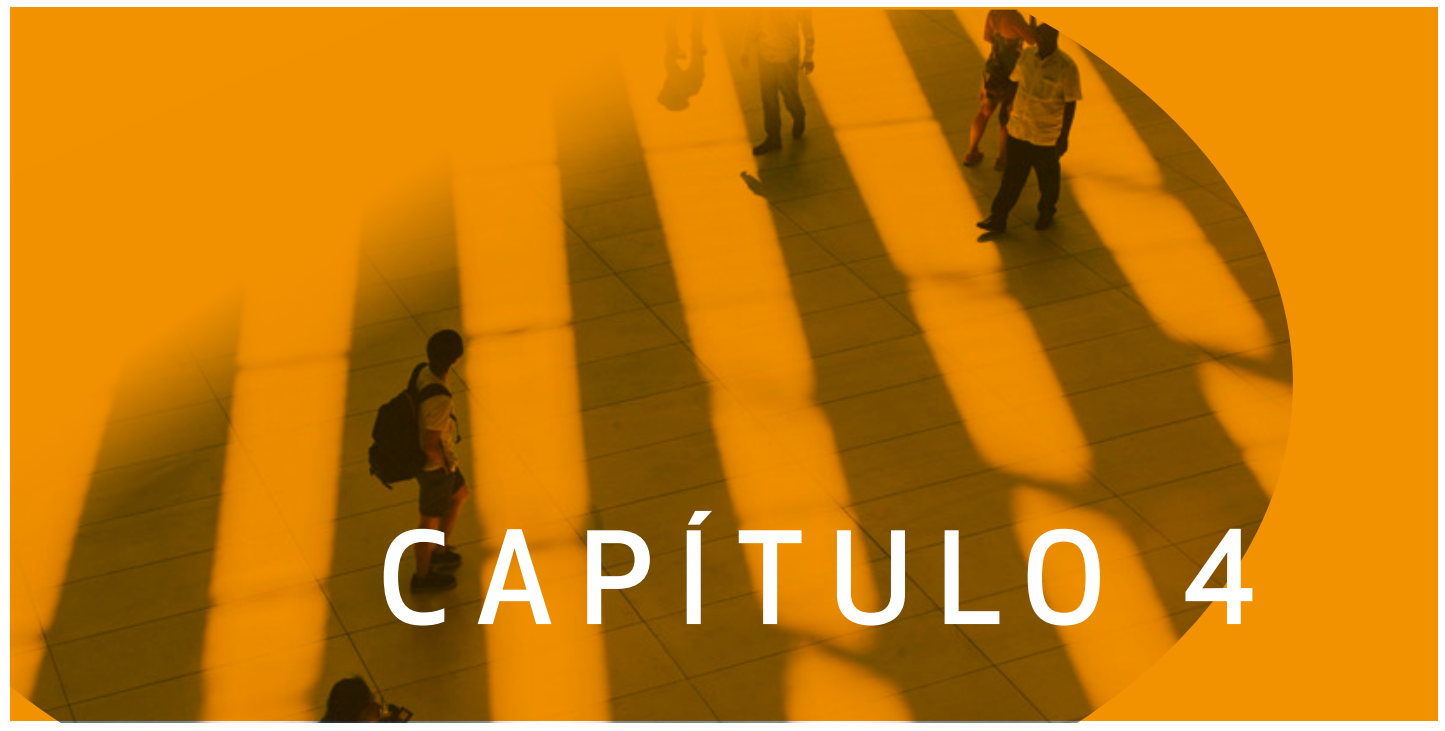





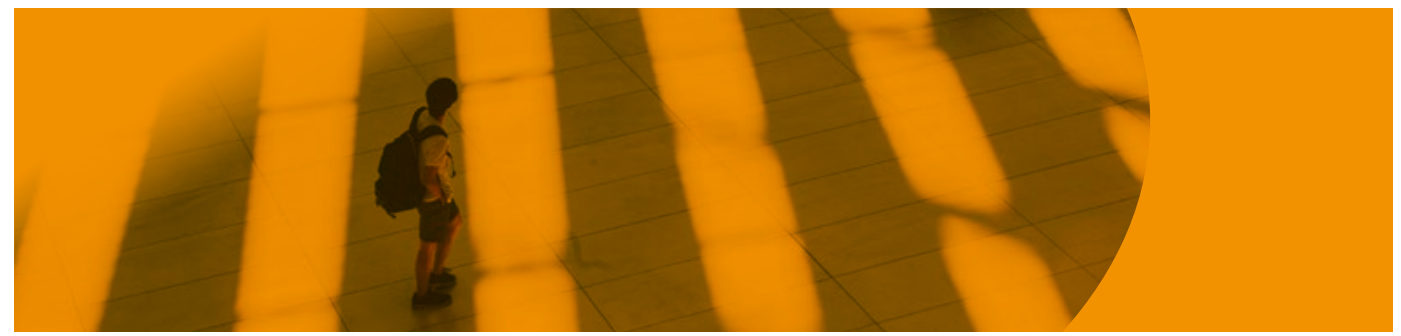

\section{Tendencias para la educación contable}

\section{Martha Lucía Fuertes Díaz}

Doctora en Administración Gerencial (C), Universidad Benito Juárez, México. Magíster en Administración con énfasis en Finanzas Corporativas, Universidad ICESI, Colombia. Especialista en Finanzas con concentración en Finanzas Avanzadas, Universidad ICESI, Colombia. Contadora Pública, Universidad Santiago de Cali, Colombia. Docente Universidad Nacional Abierta y a Distancia, UNAD, Colombia. Investigadora principal Grupo de Investigación ilama.

Correo electrónico: martha.fuertes@unad.edu.co

ORCID: http://orcid.org/0000-0001-8468-1867

Google Scholar: https://scholar.google.com.co/citations?user=IP5s-

\section{José Obdulio Cúrvelo Hassán}

Doctor en Contabilidad, Universidad de Valencia, España. Magíster en Filosofía, Universidad Santo Tomás, Colombia. Especialista en Revisoría Fiscal y Control de Gestión, Universidad Cooperativa, Colombia. Contador público, Universidad Cooperativa, Colombia. Decano Nacional de las Facultades de Contaduría pública de la Universidad Cooperativa de Colombia. Director del Grupo de Investigación Contabilidad y Entorno Social.

Correo electrónico: jose.curvelo@ucc.edu.co 


\section{Introducción}

Identificadas las políticas públicas que regulan el desempeño de los profesionales de la contaduría pública y el estado actual de la educación contable superior en el contexto nacional e internacional, se procedió con la determinación de las necesidades del tejido empresarial, desde los diferentes sectores de la economía, y las necesidades de la población respecto de la formación profesional del contador público. Aspectos identificados que se constituyen en factores fundamentales para establecer las oportunidades para la educación contable superior demandada por el tejido empresarial y social de Colombia.

En el capítulo anterior se establecieron las oportunidades en la formación integral del contador público, el análisis para su desempeño competente en la disciplina como generador de impacto regional, con capacidad para enfrentar el actual contexto globalizado en que se desarrollan las organizaciones, y el ejercicio ético como base fundamental para su educación. Estos elementos aportan a la sociedad y al sector empresarial garantías para el desempeño del profesional que se reconoce a sí mismo como garante y generador de la confianza pública, coadyuvador para el desarrollo económico de las organizaciones, la sociedad y el país. Finalmente, se resalta la modalidad virtual y la flexibilidad en los programas de contaduría pública, que aportan al mejoramiento de la calidad de vida de la comunidad estudiantil desde varios referentes.

En el presente capítulo se establecen las tendencias para la educación contable, tantoparael contextolocal comoglobal. Sereconocesu concepcióncomociencia, su constante cambio y evolución que permite responder pertinentemente a las necesidades y exigencias generadas por los cambios y tendencias del desarrollo mercantil, económico y social.

Se analiza el contexto de desarrollo del conocimiento contable y la responsabilidad que les corresponde a las instituciones de educación superior en la formación de contadores públicos internacionales, en la oferta de programas como respuesta a las necesidades de la población, del sector empresarial, las tendencias del ejercicio profesional y las políticas públicas. 
Para ello se fundamenta la propuesta a partir de la identificación de núcleos problémicos y núcleos integradores de problemas, y su articulación con los campos de formación y las competencias, entre otros factores relevantes.

\section{Tendencia global en el horizonte de conocimiento contable}

La contabilidad y el contador público han sido sujetos de transformaciones debido a las tendencias hacia la globalización, la competitividad y las innovaciones tecnológicas. Situación que ha llevado a ampliar su función más allá del registro de la información financiera y avanzar en los retos actuales mediante el desarrollo de competencias disciplinares aplicables en contextos locales, regionales e internacionales. El contador público es agente generador de valor agregado a partir de la información financiera que gestiona para contribuir con el proceso de toma de decisiones, aporta al crecimiento y permanencia del tejido empresarial y del bienestar social.

Sin lugar a dudas, el factor coyuntural que enfrenta actualmente la contaduría pública y las instituciones de educación superior, se deriva del proceso de armonización a estándares internacionales de información financiera y de aseguramiento de la información, con los cuales se pretende que la contabilidad de todas las organizaciones en Colombia se prepare sobre bases uniformes de aplicación mundial. Esto contribuye al logro de mayores niveles de competitividad, garantiza la información financiera útil, pertinente, confiable y comparable, sobre las cuales los relacionados económicos "stakeholders" puedan apoyar su proceso de toma de decisiones, en el contexto nacional e internacional. Es para enfrentar este escenario que se debe preparar al futuro profesional.

El proceso de convergencia se establece en Colombia a partir de la Ley 1314 de 2009, que abre el camino para la adopción de las normas internacionales de contabilidad tanto para el sector privado que tiene que aplicar las Normas Internacionales de Información Financiera (NıIF) o International Financial 
Reporting Standards (IFRS), como las Normas Internacionales de Contabilidad para el sector público (NıcSP).

Es importante señalar que, en Colombia, la contabilidad se venía aplicando de acuerdo con los Principios de Contabilidad Generalmente Aceptados (PCGA), los cuales estaban amparados por el Decreto 2649 de 1993, de aceptación en Colombia, pero no de aceptación internacional. Este aspecto limitaba a los usuarios de la información financiera en el actual contexto globalizado, de auge de tratados internacionales para el libre comercio, de desarrollo de los mercados de capitales mundiales, para desarrollar relaciones comerciales y económicas, generando un efecto limitante en los estándares de competitividad de las organizaciones.

En este escenario se establece el proceso de convergencia desde los PccA a las IFRS a partir de la Ley 1314 de 2009. Las IFRS O NIIF han tenido gran acogida en el ámbito mundial, y como lo señala Ledezma (2015), han sido incorporadas en 130 países. En la tabla 12 se ilustra el avance en cuanto al número de países y regiones del mundo que han realizado la convergencia a los estándares internacionales, o que se encuentran en ese proceso. 
Tabla 12. Adopción de las NıIF a nivel mundial, 2015.

\begin{tabular}{|c|c|c|c|}
\hline \multicolumn{4}{|c|}{ PAÍSES ENCUESTADOS } \\
\hline Regiones & En la región & $\begin{array}{c}\text { Aplicación de NIF } \\
\text { en todas o la mayoría } \\
\text { de entidades que cotizan } \\
\text { en Bolsa }\end{array}$ & $\begin{array}{l}\text { \% Países que requieren } \\
\text { NIF / Total de la región }\end{array}$ \\
\hline Europa & 43 & 42 & $98 \%$ \\
\hline África & 19 & 15 & $79 \%$ \\
\hline Oriente Medio & 9 & 8 & $89 \%$ \\
\hline Asia - Oceanía & 32 & 24 & $75 \%$ \\
\hline América & 37 & 27 & $98 \%$ \\
\hline TOTAL & 140 & 116 & $83 \%$ \\
\hline$\%$ & $98 \%$ & $83 \%$ & \\
\hline
\end{tabular}

Fuente: Ledezma, 2015

Históricamente, mientras en Colombia se aplicaba un marco normativo contable local, en el año 2001 se creaba en Londres la Junta de Normas Internacionales de Contabilidad (International Accounting Standards Board, IASB), como organismo independiente y de carácter privado que desarroIla y aprueba las normas Internacionales de Información Financiera (NIIF) o International Financial Reporting Standards (IFRS) (Ledezma, 2015).

El IASB se creó en reemplazo del Comité de Normas Internacionales de Contabilidad o International Accounting Standards Committee (IASC), quien en su momento emitió las Normas Internacionales de Contabilidad o International Accounting Standards (IAS). 


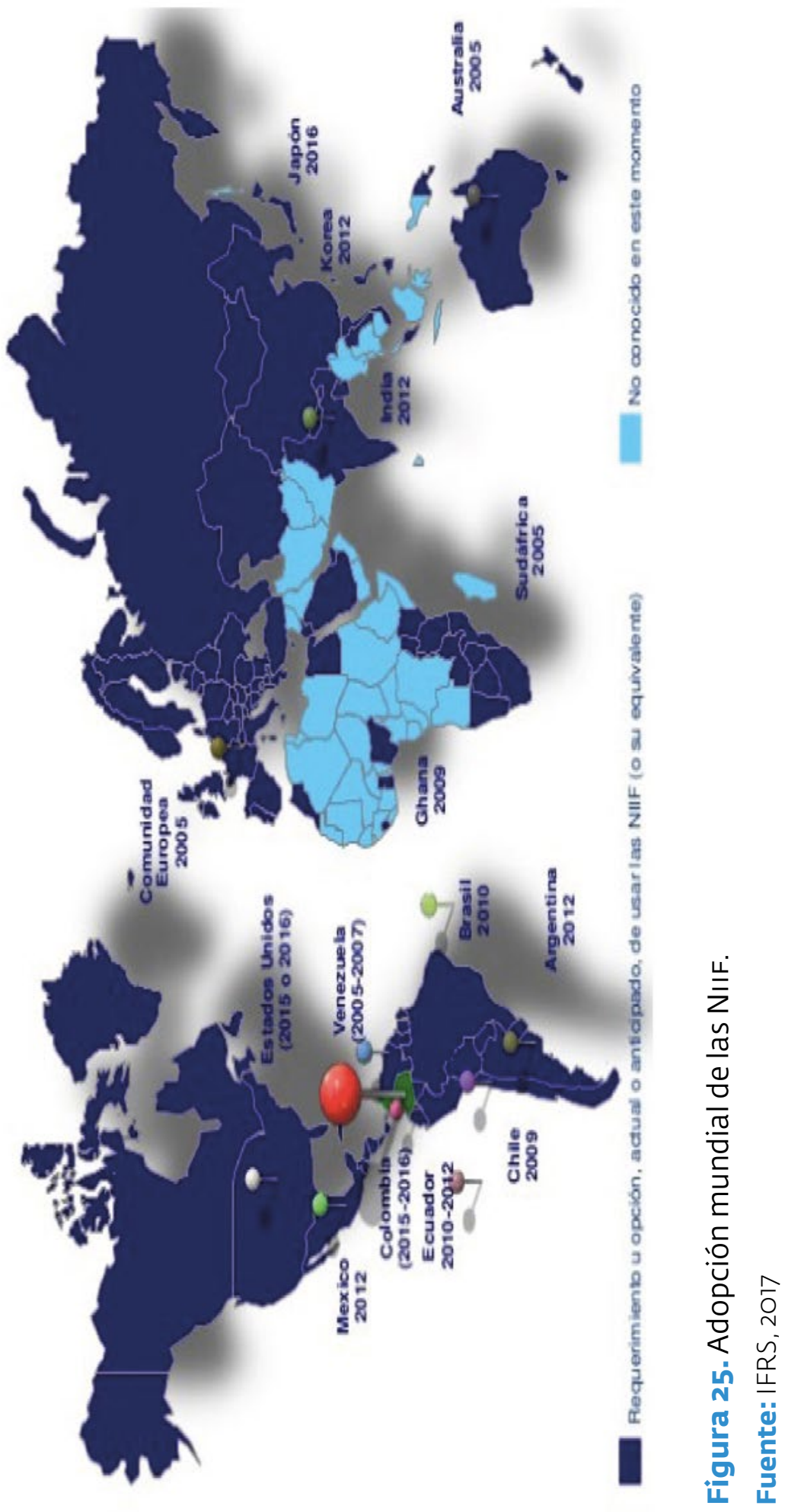


De acuerdo con este avance en el ámbito mundial, Colombia, desde la generación de la Ley 1314 en el año 2009, se encuentra en un momento coyuntural producto del proceso de armonización y adopción de estándares internacionales a partir de las Nır para todo tipo de organizaciones, grandes, medianas, pequeñas y microempresas, para lo cual se establecen tres grupos para el proceso de convergencia a estándares internacionales.

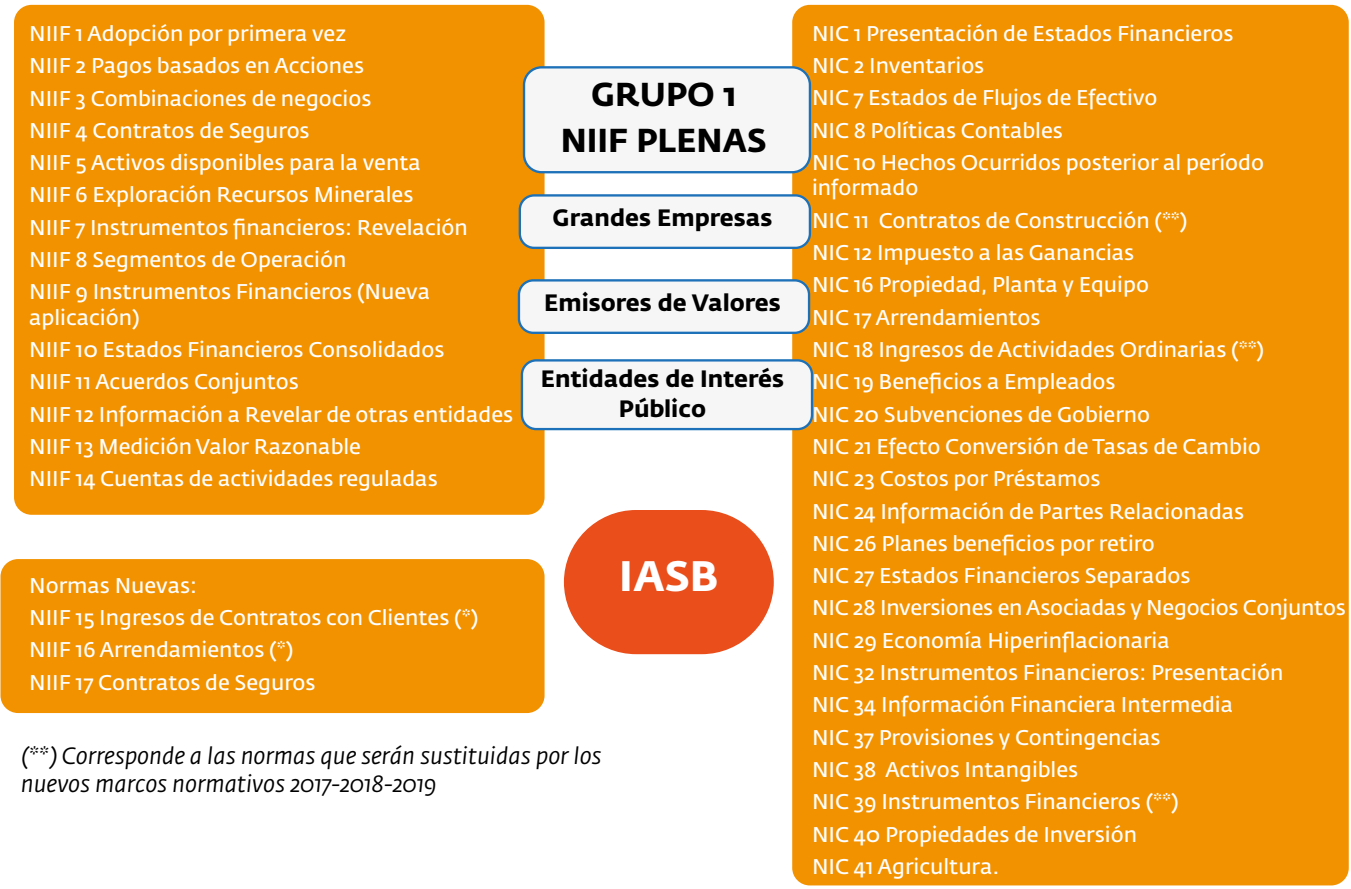

Figura 26. NIIF Plenas, Grupo 1

Fuente: elaboración propia

Para el grupo 1, que corresponde al de grandes empresas, se sustenta la aplicación de las Normas Internacionales de Contabilidad basadas en los estándares internacionales denominadas como NiIF Plenas en Colombia, que se detallan en la figura 26. 


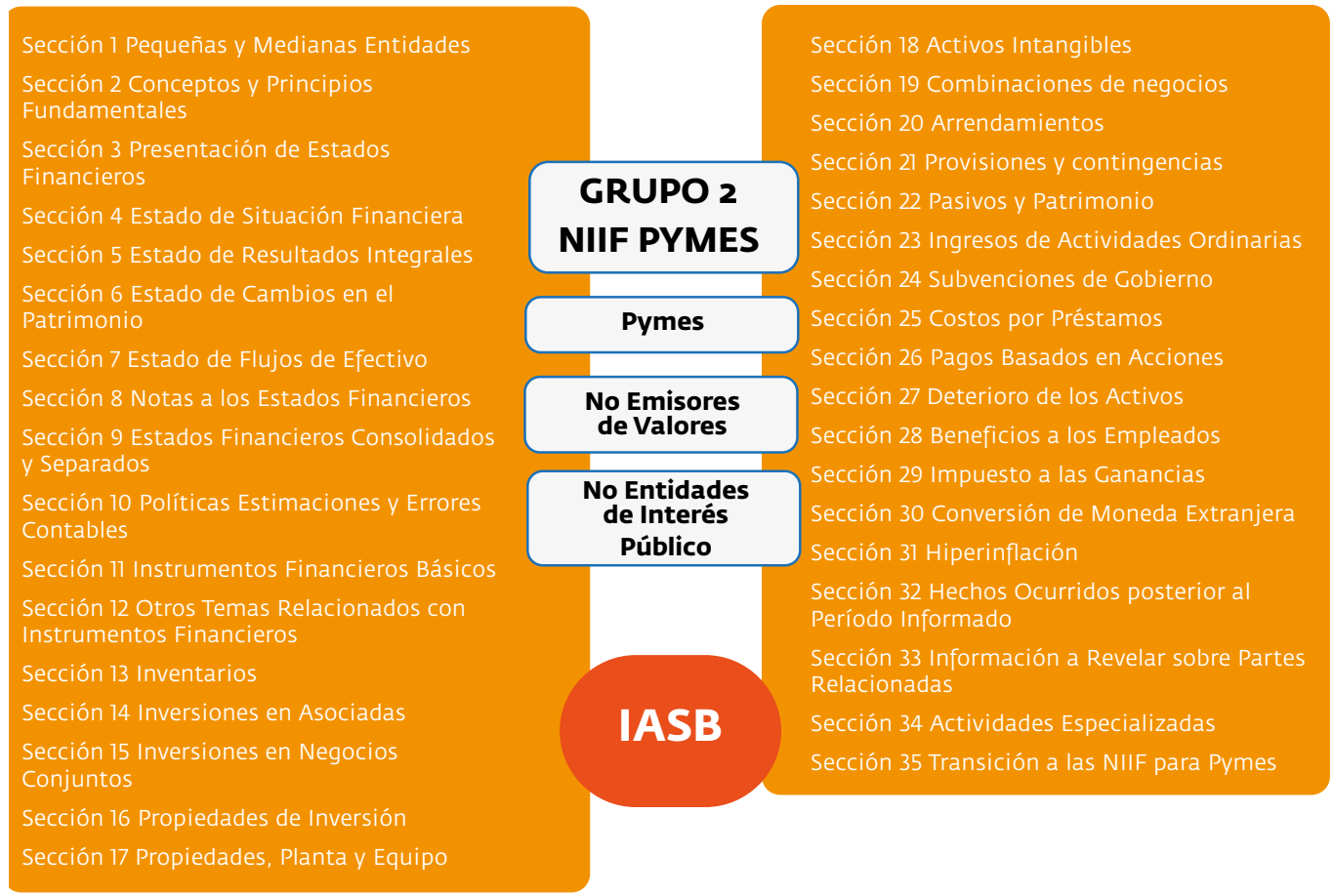

Figura 27. NIIF Pymes.

Fuente: elaboración propia

Para las pequeñas y medianas empresas se establece la aplicación de normas internacionales con base en la NıIF para Pymes, que se detallan en la figura 27. Para el caso de las microempresas se establece el marco normativo para microempresas aplicable en Colombia.

Según el Direccionamiento Estratégico emitido por el Consejo Técnico de la Contaduría Pública (СTCP), como autoridad de normalización técnica de las normas contables de información financiera y de aseguramiento de la información a aplicarse en Colombia, esta entidad toma como referente los marcos conceptuales que se ilustran en la tabla 13, para la aplicación de la contabilidad de Colombia con base en los estándares internacionales. 
Tabla 13. Normas de información financiera aplicables en Colombia, СTCP.

\begin{tabular}{|c|c|c|}
\hline $\begin{array}{l}\text { Normas de } \\
\text { Información } \\
\text { Financiera -NIF }\end{array}$ & $\begin{array}{c}\text { Normas de Aseguramiento } \\
\text { de la Información } \\
\text {-NAI }\end{array}$ & $\begin{array}{c}\text { Otras Normas } \\
\text { de Información Financiera } \\
\text {-ONI }\end{array}$ \\
\hline 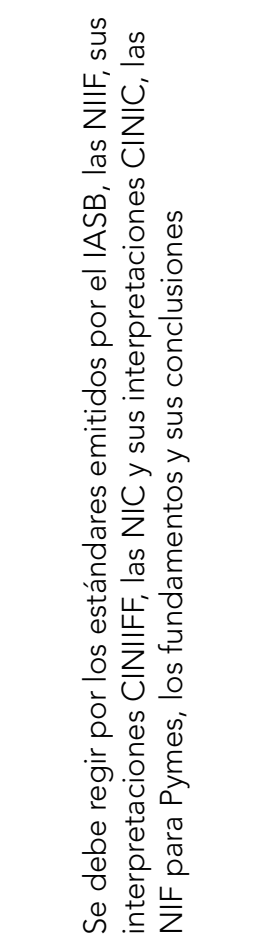 & $\begin{array}{l}\text { Se debe regir por los estándares } \\
\text { emitidos por EL IASB a saber: } \\
\text { - Estándares Internacionales } \\
\text { de Auditoría -ISA } \\
\text { - Estándares Internacionales sobre } \\
\text { Trabajos de Revisión Limitada -ISAE } \\
\text { - Estándares Internacionales sobre } \\
\text { Servicios Relacionados ISRS } \\
\text { - Estándares Internacionales de } \\
\text { Control de Calidad para los } \\
\text { servicios incluidos en los estándares } \\
\text { del IAASB-ISOC. } \\
\text { Además: } \\
\text { - Los Estándares Internacionales } \\
\text { sobre Prácticas de Auditoría } \\
\text {-IAPS emitidos por el comité } \\
\text { Internacional de Prácticas de } \\
\text { Auditoria -IAPC } \\
\text { - El código de Ética para Contadores } \\
\text { Profesionales emitido por la Junta } \\
\text { de Estándares Internacionales de } \\
\text { Ética para Contadores -IESBA. }\end{array}$ & $\begin{array}{l}\text { Referentes al sistema } \\
\text { documental contable, incluye } \\
\text { soportes, comprobantes y } \\
\text { libros, como los informes } \\
\text { de gestión y la información } \\
\text { contable, registro electrónico } \\
\text { de libros, depósito electrónico } \\
\text { de información, reporte de } \\
\text { información mediante XBRL, } \\
\text { entre otros. }\end{array}$ \\
\hline
\end{tabular}

Fuente: Direccionamiento Estratégico CTCP, 2015

En este escenario, el sector educativo adquiere un papel protagónico por la responabilidad que le corresponde en la formación de los profesionales de la contaduría pública capaces de gestionar, analizar y controlar la información financiera que generan las organizaciones, de acuerdo con los marcos conceptuales internacionales y de aseguramiento de la información. Lo anterior, tanto para la gestión de la información como para comprender la dinámica en que se desarrollan negocios, para así aportar al fortalecimiento, crecimiento y sostenibilidad de las organizaciones, observando los prinicipios éticos que desde la "disciplina" y del "ser" que exige esta profesión como garantes de la confianza pública para su desempeño, ya no con impacto local sino internacional y global. 
A partir de la expedición de la Ley 1314 de 2009 se generaron políticas públicas a través de diferentes decretos que debieron ser modificados y posteriormente derogados. Por lo cual se presenta, en la figura 28 , la última normativa expedida hasta la que se encuentra vigente actualmente.

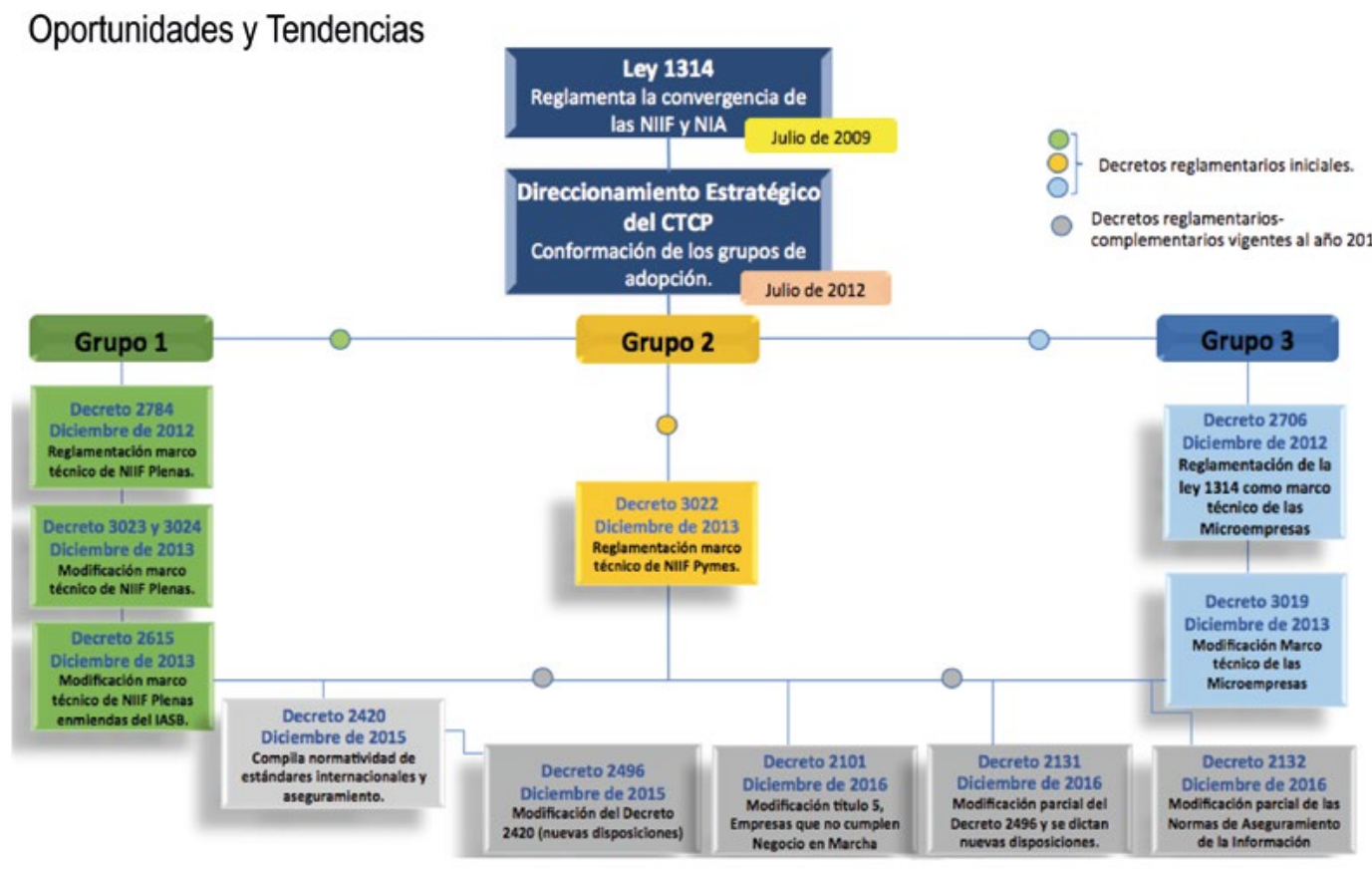

Figura 28. Política pública relacionada con el conocimiento contable.

Fuente: elaboración propia

Los temas abordados en los pàrrafos anteriores demanda de las instituciones de educación superior, en sus diseños curriculares, su aplicación para el logro de programas de contaduría pública internacional, como lo exigen las tendencias mercantiles, económicas, disciplinares y sociales. 


\section{Tendencia hacia la modalidad virtual para los programas de contaduría pública}

Actualmente, y de acuerdo con el estudio Tendencia, oportunidad y estrategias en la formación de profesionales de contaduría en modalidad presencial realizado por el Observatorio de la Universidad Colombiana (Cardozo, 2017), se reconoce, para programas de contaduría pública, que los cambios coyunturales que se han dado a partir de la implementación obligatoria a estándares internacionales y la creciente exigencia que en materia fiscal debe asumir toda organización, han convertido esta profesión en una de las más atractivas a elegir en los últimos años.

En este estudio se analizan variables como el precio de matrícula, la modalidad de formación, la duración del programa, la financiación, la ubicación geográfica y la acreditación. Esto le permite establecen cuál de ellas tiene mayor incidencia en la motivación de los aspirantes a estudiar un programa de contaduría pública y la transferencia de la modalidad presencial a la no presencial y virtual.

Tabla 14. Estudiantes del país inscritos contra admitidos, programa contaduría pública.

\begin{tabular}{lcccccc}
\multirow{2}{*}{\begin{tabular}{l} 
Inscritos \\
\cline { 2 - 7 }
\end{tabular}} & $\begin{array}{c}\text { No. } \\
\text { Estudiantes }\end{array}$ & $\%$ & $\begin{array}{c}\text { No. } \\
\text { Estudiantes }\end{array}$ & $\%$ & $\begin{array}{c}\text { No. } \\
\text { Estudiantes }\end{array}$ & $\%$ \\
\hline Bogotá & 17.251 & $34 \%$ & 14.241 & $43 \%$ & 3.010 & $17 \%$ \\
\hline Cundinamarca & 903 & $2 \%$ & 645 & $4.5 \%$ & 258 & $2 \%$ \\
\hline Nacional & 50.139 & $100 \%$ & 32.965 & $100 \%$ & 17.251 & $100 \%$ \\
\hline
\end{tabular}

Fuente: Cardozo, 2017. 
Como lo señala Cardozo (2017), para el año 2015 se inscribieron a programas de contaduría pública 50.139 aspirantes a nivel nacional, de los cuales 32.695 fueron admitidos, lo que demuestra una tasa de efectividad del $65 \%$ en Colombia. Bogotá concentra el 34 \% de los aspirantes inscritos al programa, con 17.251, de los cuales fueron admitidos 14.241 estudiantes, lo que implica una efectividad del $43 \%$, muy por debajo del promedio nacional, como se aprecia en la tabla 14.

En el estudio se toman las diez universidades de la ciudad de Bogotá con mayor número de estudiantes matriculados en el programa de contaduría pública, que agrupan un total de 9.226 estudiantes, donde se identifica la modalidad, duración y precio para el programa como variables a estudiar.

Del total de 9.226 estudiantes que se matricularon en Bogotá al programa de contaduría pública, 5.686 eligieron la modalidad no presencial, lo cual es equivalente al 61,6\%, mientras que el 38,4\%, con 3540 estudiantes, optaron por la modalidad presencial. Este aspecto evidencia claramente un cambio de tendencia en la elección de la modalidad de estudio, la cual está viviendo la transición de la presencialidad a la modalidad a distancia tradicional y la modalidad virtual (Cardozo, 2017).

En la tabla 15 se aprecia que, de 5.686 estudiantes que se matricularon en la modalidad no presencial, 2.518 estudiantes eligieron la modalidad virtual lo que equivale al $44 \%$. Estos aspectos demuestran, en los últimos años, el cambio de tendencia en la predilección de programas en modalidad virtual en este campo de conocimiento, dado que implica mayor eficiencia en el manejo del tiempo de los estudiantes. Esta modalidad aporta significativamente al mejoramiento de su calidad de vida, al mejoramiento de sus competencias en las TIC's y, en lo económico, los precios de matrícula son mucho más bajos que los que se ofrecen en programas presenciales y en distancia tradicional. 
Tabla 15. Las diez universidades con mayor número de estudiantes admitidos, año 2015.

\begin{tabular}{|c|c|c|c|c|c|c|}
\hline : & $\begin{array}{l}\text { Institución } \\
\text { de Educación Superior (IES) }\end{array}$ & $\begin{array}{l}\frac{0}{0} \\
\frac{\pi}{0} \\
\frac{0}{0} \\
\frac{0}{10} \\
\frac{0}{8} \\
\frac{0}{2}\end{array}$ & ¿ & 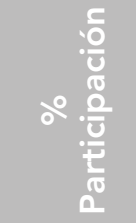 & $\begin{array}{l}\frac{6}{3} \\
\frac{0}{3} \\
\frac{10}{5}\end{array}$ & $\frac{0}{\frac{\pi}{3}} \frac{u}{\frac{u}{2}}$ \\
\hline \multirow{3}{*}{1} & \multirow{3}{*}{$\begin{array}{l}\text { COPORACION UNIVERSITARIA } \\
\text { MINUTO DE DIOS -UNIMINUTO }\end{array}$} & Distancia $(T)^{\star}$ & 2.999 & $85,4 \%$ & 9 & 1.534 .000 \\
\hline & & Presencial & 513 & $14,6 \%$ & 9 & 2.542 .000 \\
\hline & & Total & 3.512 & $100,0 \%$ & & \\
\hline \multirow{3}{*}{2} & \multirow{3}{*}{ POLITÉCNICO GRANCOLOMBIANO } & Virtual & 1.554 & $79,7 \%$ & 9 & 1.499 .000 \\
\hline & & Presencial & 396 & $20,3 \%$ & 9 & 3.004 .000 \\
\hline & & Total & 1.950 & $100,0 \%$ & & \\
\hline \multirow{3}{*}{3} & \multirow{3}{*}{$\begin{array}{l}\text { FUNDACIÓN UNIVERSITARIA } \\
\text { DEL ÁREA ANDINA }\end{array}$} & Virtual & 964 & $61,2 \%$ & 9 & 1.890 .000 \\
\hline & & Presencial & 611 & $38,8 \%$ & 9 & 2.600 .000 \\
\hline & & Total & 1.575 & $100,0 \%$ & & \\
\hline \multirow{3}{*}{4} & \multirow{3}{*}{$\begin{array}{l}\text { UNIVERSIDAD MILITAR NUEVA } \\
\text { GRANADA }\end{array}$} & Distancia $(T)^{\star}$ & 169 & $80,5 \%$ & 9 & 3.540 .000 \\
\hline & & Presencial & 41 & $19,5 \%$ & 9 & 3.725 .000 \\
\hline & & Total & 210 & $100,0 \%$ & & \\
\hline 5 & $\begin{array}{l}\text { UNIVERSIDAD COOPERATIVA DE } \\
\text { COLOMBIA }\end{array}$ & Presencial & 621 & $100 \%$ & 8 & 2.151 .904 \\
\hline 6 & $\begin{array}{l}\text { FUNDACIÓN UNIVERSITARIA LOS } \\
\text { LIBERTADORES }\end{array}$ & Presencial & 483 & $100 \%$ & 8 & 2.701 .000 \\
\hline 7 & UNIVERSIDAD CENTRAL & Presencial & 341 & $100 \%$ & 10 & 3.664 .920 \\
\hline 8 & UNIVERSIDAD AGUSTINIANA & Presencial & 236 & $100 \%$ & 9 & 2.663 .000 \\
\hline 9 & UNIVERSIDAD SANTO TOMAS & Presencial & 195 & $100 \%$ & 10 & 3.776 .000 \\
\hline \multirow[t]{4}{*}{10} & CORPORACIÓN IBEROAMERICANA & Presencial & 103 & $100 \%$ & 10 & 2.223 .000 \\
\hline & \multirow{3}{*}{$\begin{array}{l}\text { TOTAL } 10 \text { UNIVERSIDADES } \\
\text { EN BOGOTÁ }\end{array}$} & No Presencial & 5.686 & $61,6 \%$ & & \\
\hline & & Presencial & 3.540 & $38,4 \%$ & & \\
\hline & & Total & 9.226 & $100,0 \%$ & & \\
\hline
\end{tabular}

Fuente: Cardozo, 2017.

La tendencia hacia la educación virtual que demanda la comunidad estudiantil del país, amparada en el desarrollo tecnológico que aporta al mejoramiento de su calidad de vida, a la cobertura educativa impactando hasta las regiones 
más apartadas de la geografía nacional donde la educación tradicional no hace presencia, el acceso al conocimiento y a las redes de conocimiento en tiempo real, en cualquier momento y lugar, explican cómo entre los años 2014 a 2016 se han creado once nuevos programas de contaduría pública en modalidad virtual.

\section{Tendencia: núcleos problémicos pertinentes para la educación contable}

La lógica de las ciencias sociales contemporaneas, como bien lo presenta Habermas (1995), presuponen entender integralmente los problemas que han movido las relaciones humanas, y el reconocimiento a través del lenguaje, de las formas de vida no humana y considerar sujetos a todos aquellos que generen discursos.

Con la teoría de la acción comunicativa, en el cual Habermas recoge los hitos de un permanente proceso de auto-aclaración, donde devela las corrientes fundamentales de la filosofía y la teoría sociológica contemporáneas, y da forma a las nuevas lógicas de las ciencias sociales. Esta consideración implica el conocimiento por el giro lingüístico que considera sujetos a todos aquellos capaces de emitir discurso, así mismo, adscribe la ética a una tradición netamente analítica, en tanto acuerdo que establece determinada comunidad lingüística sobre las actuaciones.

Adicional a lo anterior Vessuri, et al. (2014), al igual que Lux y Pérez (2017), plantean que la comprensión de las ciencias sociales, según los estándares de divulgación social del conocimiento, han conllevado a que los objetos de dichas ciencias se centren en problemas más de carácter regional y no en la resolución de problemas relevantes en los contextos locales. Este planteamiento retoma la manera de entender las ciencias sociales, abordado en el Informe Mundial de la Ciencia de UnEsCo de 1998: "el progreso en las ciencias consiste, en parte, en plantearse las viejas preguntas de manera más lúcida y penetrante”.

En dicho contexto, la contabilidad, como ciencia eminentemente social, deberá atender tanto en su desarrollo técnico como en la construcción epistemológica, 
respuestas a las demandas de la vida cotidiana y a los avances en materia científica y tecnológica. Estas manifestaciones se adscriben a la cultura, donde "el conocimiento constituye el principal activo" ha llevado a que la contabilidad tome un rol protagónico al favorecer, de manera científica, la determinación y el control de todos los procesos de producción de la vida moderna, la generación y distribución de la riqueza, de igual forma, ser garante de la confianza en el ámbito global (Craig, et al., 2014).

Esteaportea la eficacia de los negocios está también aliado con el podereconómico y político de las empresas y del Estado, de igual manera a las reivindicaciones de las sociedades en materia de control social y rendición de cuentas (Gray, et al, 2014; Rhodes, 2017; Gray, et al., 1996).

Los hechos antes enunciados han conllevado a un desarrollo en materia de investigación contable, lo que redunda en sus construcciones epistemológicas. También se han generado avances técnicos, con gran incidencia en el ámbito normativo y de la regulación.

Siendo así, la contabilidad y la contaduría pública están configuradas por las relaciones con la vida en sociedad, tal como lo evidencian los trabajos de Walks (2016), Jeacle y Miller (2016) y Joseph (2014). Desde estas perspectivas, la contabilidad como lenguaje de los negocios, supone contextos, condiciones de calidad y expresiones que aseguran un clima ético de los negocios, la salvaguarda del interés público, la confianza entre los contratantes y un adecuado funcionamiento socioeconómico.

Desde esta perspectiva, la contabilidad asume un rol protagónico, como saber científico, en tanto configura el devenir de los negocios (Tua-Pereda: 2012). Es decir, permite la comprensión de los acontecimientos sociales en los que estos se realizan y la manera como sus actores logran establecer nuevas formas de relaciones.

Según Rojas y Ospina (2011) afirman que el problema del conocimiento histórico y social que ha liderado la carencia teórica en el seno de la problemática contable, 
está siendo fuertemente cuestionado. Lo anterior está asociado a las nuevas lógicas que subyacen en el pensamiento científico de la sociedad del siglo xxı (Castells, 2005), el cual permite comprender que el conocimiento se ha constituido en un bien. Por ello, los sistemas de administración histórico no necesariamente responden a sus formas de gestión y, lo que es más complejo, comprender la manera cómo evidenciar su función en la generación, acumulación y redistribución de la riqueza, aspectos que constituyen un imperativo en la contabilidad.

Tedesco (2017) responde a estas nuevas realidades, evidenciando que tanto el Estado como las empresas han desarrollado nuevos procesos de relegitimación, mediante una nueva noción institucional de la escuela, abriéndola a los requerimientos de la sociedad y redefiniendo la universidad a partir del desarrollo de la empresa, con la necesidad de un conocimiento tanto tecnológico como científico. Estos aspectos generan en el desarrollo científico de la contabilidad nuevas formas de comprender las formas de llegar al conocimiento y la forma de dimensionar el alcance de sus objetos.

La captura de estos fenómenos y su reporte a la sociedad, a través de la contabilidad, evidencia desarrollos científicos importantes de esta disciplina, no obstante, las implicaciones de vincular el conocimiento como un activo esencial de la empresa sigue constituyéndose en uno de los principales retos para la contabilidad contemporánea.

Es de precisar que dentro de estas configuraciones se encuentran nuevas categorías en los negocios, como la innovación social, el emprendimiento solidario (Irigoyen, 2016) e incluso el rol político de las organizaciones (Coraggio, 2004). Por otra parte, en cuanto a las formas empresariales, se han generado redes mecánicas de distribución, así mismo formas solidarias de negocios que se centran en el comercio justo (Coraggio, 2015) y en lógicas contrapuestas a la economía de mercado (Beúnza, 1996). Muy a pesar de estas nuevas manifestaciones, la contabilidad cumple al interior de estas formas de organización el lenguaje de los negocios, poniéndolos en dialogo con las lógicas de economía de mercado y de empresas lucrativas. 
Atendiendo a lo anterior, es evidente que la sociedad de finales del siglo xx y lo queva corrido del sigloxxı ha generado nuevos paradigmas para la contabilidad. Como resultado de lo anterior, Huang (2014) planteará que los problemas, en tanto a captura, representación y divulgación de la contabilidad, están en la medición, aspecto que se contrapone a la noción clásica alrededor del derecho y a una economía centrada en la riqueza. También, entrará en estos desafíos el cambio en el paradigma del poder político y el nuevo rol que asume la sociedad en la autodeterminación.

De acuerdo con las anteriores reflexiones, las propuestas curriculares para los programas de contaduría pública exigen una metodología explicativa e investigativa para la identificación de las causas críticas que generan los problemas que se deben resolver, mediante una planificación inteligente, prospectiva y situacional (Unad, 2011).

El proceso descrito en los primeros capítulos del libro conducen la formulación de los núcleos problémicos para el programa de contaduría pública, como fuente generadora de conocimiento de las demandas y necesidades en el contexto global, nacional, regional, local, disciplinar, la academia y el sector productivo, que confluyen en el núcleo integrador de problemas (NIP), a partir de la concepción del carácter eminente social del conocimiento contable. A continuación, se proponen los Núcleos Problémicos (NP) identificados en este proceso, acordes con los anteriores postulados, los cuales se ilustran en la figura 26.

NP1: Competencias profesionales de la disciplina contable y su formación integral frente a los retos en contextos globalizados y cambiantes.

NP2: Desarrollo competitivo, sostenible del tejido empresarial, desde los contextos regionales con enfoque global.

NP3: Responsabilidad social y ambiental en la búsqueda de soluciones responsables, creativas e innovadoras para la transformación social. 
Diseño Curricular

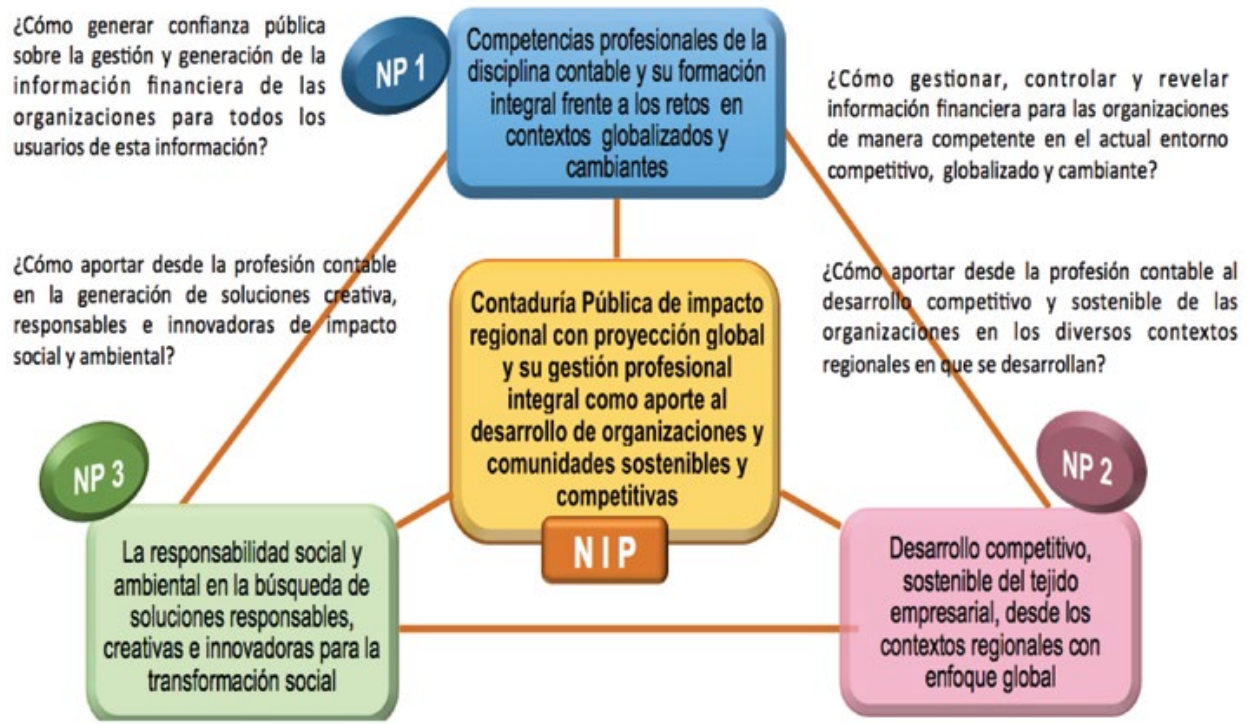

Figura 29. Núcleos problémicos y Núcleo Integrador de Problemas (NIP).

Fuente: elaboración propia

A partir de los NP establecidos, se consolida la propuesta en el NIP que enmarca el discurso disciplinar, para la propuesta de programas de contaduría pública: NIP: Contaduría pública de impacto regional con proyección global y su gestión profesional integral como aporte al desarrollo de organizaciones y comunidades sostenibles y competitivas.

A partir de los núcleos problémicos propuestos se desarrolla la propuesta curricular del programa, el cual tiende a la formación de un profesional integral, competente en su disciplina y formado bajo estándares internacionales para la educación contable, preparado para impactar significativamente las organizaciones del país y responder competentemente ante los retos del actual contexto globalizado en que se desarrollan. Generador de la confianza pública a partir de su desempeño ético y socialmente responsable, comprometido mediante la acción investigativa con la búsqueda de soluciones creativas e innovadoras que le permitan aportar soluciones a las problemáticas que enfrentan las organizaciones, las comunidades y el medio ambiente. 
Los campos de formación en que se distribuye la red de cursos deben orientarse a la formación básica, disciplinar y complementaria. La formación básica se concibe, desde la UNAD (2011), como escenario formativo que busca generar competencias derivadas del diálogo entre saberes y disciplinas, el pensamiento científico universal en el entorno de la sociedad global y colombiana.

El campo de formación disciplinar se orienta a la gestión de conocimiento propio de las áreas que integran un programa en particular para desarrollar los enfoques teóricos, metodológicos y las competencias. El campo de formación complementaria está enfocado a coadyuvar el proceso de formación integral del estudiante, desde contextos diferentes a los disciplinares como el físico-ambiental, psicosocial, formación estética, fisiológica y espiritual. A estos campos de formación se debe sumar aquel que posibilita la construcción de la identidad institucional y la apropiación del aprendizaje autónomo, significativo y colaborativo, en la modalidad de educación virtual.

\section{Tendencia en las competencias para la educación contable virtual}

La UNAD en su Proyecto Académico Pedagógico Solidario definela construcción de competencias como la movilización de recursos que realiza una persona para ejecutar una acción eficazmente. También formulan las finalidades inmediatas del programa de formación, los objetivos de aprendizaje y orientan el proceso de evaluación, que incorpora varios tipos de competencias para la educación contable virtual: comunicativas, solidarias, transversales y específicas.

\section{Competencias comunicativas}

Según la UNAD (2011), las competencias comunicativas establecen procesos de comunicación asertiva, en una relación de complemento con el uso pedagógico de los artefactos o equipamiento tecnológico -hardware y software- facilitando la gestión de la información en términos de buscar, seleccionar y compartir información relevante que potencialice su proceso 
formativo. Se basa en la propuesta del ICFES con tres competencias básicas: interpretativa, propositiva y argumentativa; que se establecen en los diversos ambientes de aprendizaje y determinan en gran parte el éxito formativo en la modalidad abierta y a distancia.

Las tecnologías de la información y la comunicación presentes en los ambientes virtuales de aprendizaje aportan al proceso formativo elementos transformadores a nivel comunicativo. El flujo permanente y en diferentes niveles de la comunicación, exige al estudiante un dominio de la competencia comunicativa específica. Dado el alto nivel comunicativo escrito y de lectura que se establece en los ambientes virtuales y los diversos flujos comunicativos allí presentes, la interpretación de textos, códigos e intenciones comunicativas resulta fundamental. Así mismo, la necesidad de concretar ideas, construir hipótesis y debatir (UNAD, 2011).

Finalmente, el principio de construcción colaborativa del conocimiento, presente en los entornos virtuales de aprendizaje, requiere el consenso y la deliberación, es decir, la capacidad para argumentar las propias ideas y contrargumentar con otras.

\section{Competencias solidarias}

La UNAD (2011) establece las competencias solidarias orientadas a contribuir con la interpretación y transformación de las dinámicas comunitarias, para promover el desarrollo social como resultado de cambios en las estructuras de poder y una amplia participación social de la población en la construcción de una forma de vida más justa, digna. Concibe una dimensión de futuro en diferentes situaciones y escenarios que se asumen como retos y oportunidades para construir nuevas alternativas mediante un espacio de diálogo y reflexión.

Considera las exigencias pedagógicas, tecnológicas, sociales y humanísticas de la modalidad virtual, para definir el perfil del estudiante como el de un líder comunitario, ético y solidario, formado bajo estándares de calidad nacional e internacional, comprometido con el desarrollo de su región y con las más al- 
tas competencias cognitivas, socioafectivas, interlingüísticas, interculturales, científicas, tecnológicas e investigativas.

\section{Competencias transversales}

Son requeridas para desempeñarse en ambientes virtuales de aprendizaje, y son: la competencia digital, la ciudadana y la competencia para la autogestión del conocimiento (UNAD, 2011).

La UNAD expresa una dimensión de futuro en diferentes situaciones y escenarios asumidos como retos y oportunidades para construir nuevas alternativas.

Caracterizan las exigencias pedagógicas, tecnológicas, sociales y humanísticas de la modalidad abierta y a distancia, para definir el perfil del estudiante como líder comunitario, ético y solidario, formado bajo estándares internacional de información financiera y de aseguramiento de la información, comprometido con el desarrollo de su región y con las más altas competencias cognitivas, socio-afectivas, interlingüísticas, interculturales, científicas, tecnológicas e investigativas.

\section{Competencias específicas}

Se definen para cada programa, en este caso para el programa contaduría pública, como resultados de aprendizaje y el desarrollo del trabajo académico en las áreas de conocimiento de la profesión. Posibilitan el ejercicio responsable y adecuado de la profesión en el contexto de los núcleos problémicos que fundamentaron el diseño curricular.

Para su establecimiento se consideró la política pública vigente, las tendencias internacionales para la ciencia contable y especialmente los lineamientos establecidos en los estándares internacionales de educación contable, con las competencias propuestas desde la Federación Internacional de Contadores Públicos (IFAC) en la Norma Internacional de Formación en Contaduría (IES 3), como lo explican Dacunto y Casinelli (2009). 
Tabla 16. Competencias disciplinares IFAC.

\begin{tabular}{|c|c|}
\hline Intelectuales & $\begin{array}{l}\text { Competencia para la planeación, ejecución, supervisión y control contable y financiero y } \\
\text { tributario, de cualquier tipo de entidad nacional o internacionalmente, enfocadas hacia el } \\
\text { análisis y determinación de procesos, asignación y supervisión de recursos necesarios para } \\
\text { la ejecución, comprendiendo aspectos de recopilación, emisión y análisis de información. }\end{array}$ \\
\hline $\begin{array}{l}\text { Técnicas y } \\
\text { funcionales }\end{array}$ & $\begin{array}{l}\text { Competencia en el diseño de sistemas contables con facultades idóneas para la } \\
\text { aplicación de normas internacionales que proporcionen seguridad y fiabilidad de los } \\
\text { resultados permitiendo asegurar los procesos de emisión de estados financieros y } \\
\text { otorgando herramientas para el control fiscal, presupuestal y administrativo. }\end{array}$ \\
\hline Personales & $\begin{array}{l}\text { Competencias en el manejo de personal enfocadas a la responsabilidad dentro y fuera } \\
\text { de la organización, comprenden aspectos como integridad y comportamiento ético, } \\
\text { dinamismo y capacidad de resistencia, equilibrio entre las exigencias del trabajo y la vida, } \\
\text { conocerse así mismo y desarrollarse. }\end{array}$ \\
\hline $\begin{array}{l}\text { Interpersonales } \\
\text { y de } \\
\text { comunicación }\end{array}$ & $\begin{array}{l}\text { Competencias para el trabajo en equipo enfocadas hacia tareas realizadas por pequeños } \\
\text { grupos con responsabilidades conjuntas cuya labor es interdependiente, por lo anterior } \\
\text { comprenden aspectos como planear los equipos adecuadamente, crear un entorno de } \\
\text { equipo y manejar las dinámicas del equipo en forma adecuada. }\end{array}$ \\
\hline $\begin{array}{l}\text { Gerenciales } \\
\text { y de } \\
\text { Organización }\end{array}$ & $\begin{array}{l}\text { Competencias para la acción estratégica financiera, enfocadas hacia tareas tendientes } \\
\text { al entendimiento de la misión y los valores generales de la organización y asegurarse } \\
\text { que las acciones propias y las realizadas por quienes son dirigidos estén "sintonizadas" } \\
\text { en ese sentido, por lo anterior comprenden aspectos como entender y comprender la } \\
\text { organización y adoptar medidas estratégicas. }\end{array}$ \\
\hline
\end{tabular}

Fuente: IFAC, IES 3

\section{Tendencias para el diseño de planes de estudio de contaduría pública en modalidad virtual}

Se realiza la propuesta del plan de estudios para un programa de contaduría pública, en la modalidad virtual y con cobertura nacional, desde las áreas o campos de formación y como solución a los núcleos problémicos identificados, los cuales van a conformar las redes de cursos que constituyen el plan de estudios.

Durante el proceso de diseño del plan de estudios se espera permear los procesos académicos disciplinares para la formación integral del futuro profesional, basados en la investigación, la proyección social y la internacionalización del currículo, como elementos básicos que propician la transformación social y económica de las regiones y del país con calidad y pertinencia, que se aprecia en la figura 30. 


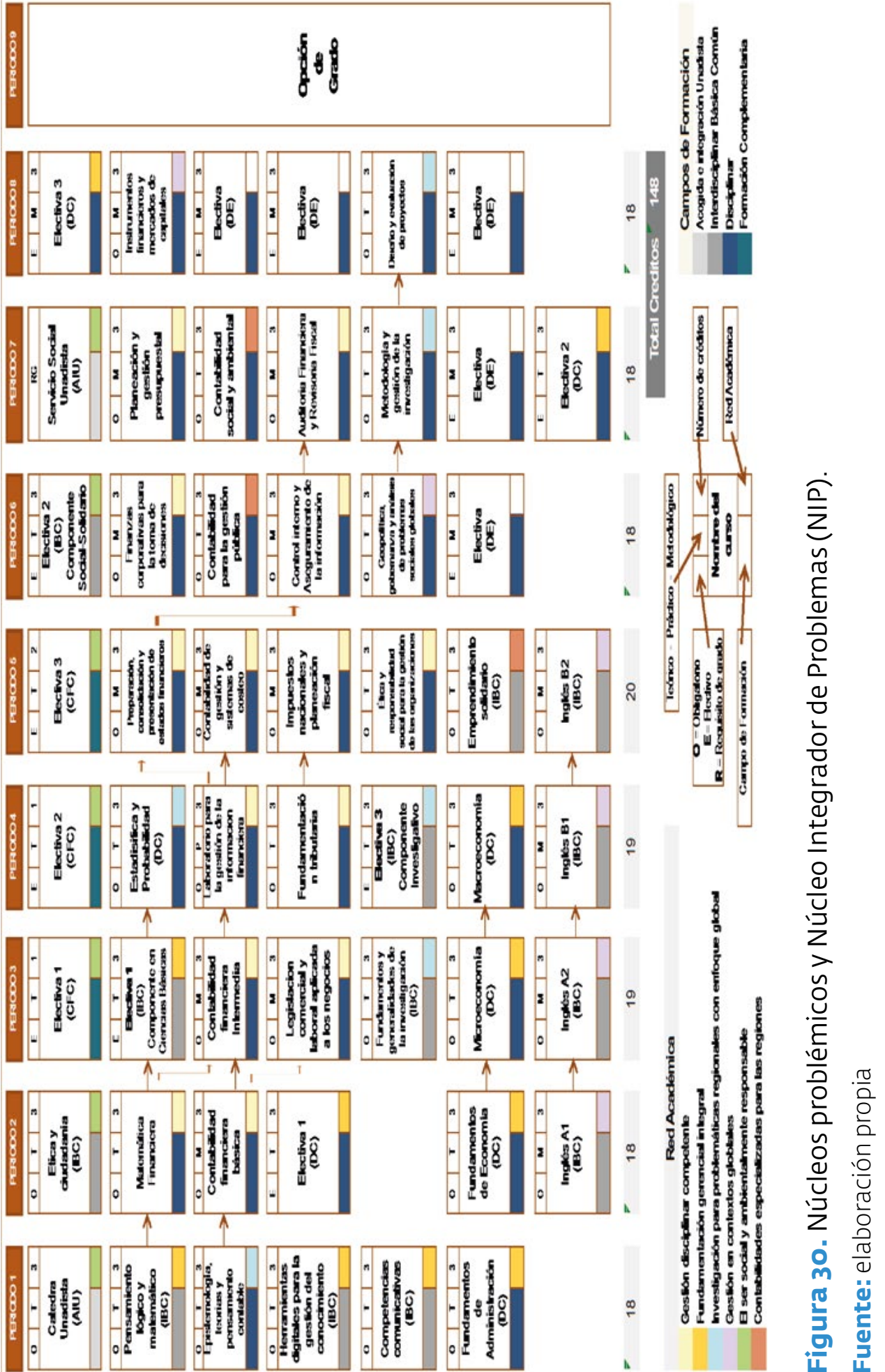




\section{Conclusiones}

En el globalizado contexto actual, el desarrollo mercantil, económico y social derivó en el acogimiento de los estándares internacionales de información financiera y de aseguramiento de la información. Así mismo, el auge y desarrollo tecnológico, con permanentes innovaciones, han aportado al desarrollo y concepción del conocimiento contable, evolucionando desde lo instrumental a lo lógico formal. Como lo señala McIntyre, Ghen y Jones (2005), esto conduce a la reflexión, al pensamiento reflexivo y crítico y de manera especial al pensamiento científico como eje dinamizador de las transformaciones profesionales y disciplinares; se constituyen en un reto para las instituciones de educación superior en Colombia responder pertinentemente a las necesidades actuales del tejido empresarial y social del país.

Las instituciones de educación superior deben avanzar creativa e innovadoramente en sus propuestas académicas para que, además de responder a la formación integral y competente del futuro contador público - consiente de la responsabilidad social y principios éticos que les corresponde como garantes de la confianza pública- puedan avanzar en el manejo eficiente del número de créditos propuestos en los planes de estudio, articulándose con la tendencia internacional para una educación contable más eficiente.

Para asegurar la pertinencia de los programas de contaduría pública, se proponen los núcleos problémicos como fuente generadora de conocimiento de las demandas y necesidades en el contexto global, nacional, regional, local, disciplinar, la academia y el sector productivo. Lo anterior confluye en el núcleo integrador de problemas, a partir del carácter eminente social del conocimiento contable.

Así, cada institución de educación superior debe orientar sus esfuerzos para contribuir en la formación de profesionales de contaduría pública capaces de actuar en el actual contexto globalizado, con sólidos conocimientos disciplinares, formación integral, socialmente responsables y elevados valores éticos, mediante el desarrollo del pensamiento crítico, la acción investigativa, la pro- 
yección social y la utilización intensiva de las tecnologías de la información y las comunicaciones. Esta formación generará contadores que aporten al desarrollo económico, social y medio ambiental de las organizaciones y de su entorno; con pertinencia regional y proyección global.

\section{Referencias}

Beúnza, J. M. I. (1996). Comunidad, red social y élites: Un análisis de la vertebración social en el Antiguo Régimen. En Elites, poder y red social: las élites del País Vasco y Navarra en la Edad Moderna (estado de la cuestión y perspectivas). Servicio de Publicaciones.

Cardozo, G. (2017). Tendencia, oportunidad y estrategias en la formación de profesionales de contaduría en modalidad presencial. Observatorio de la Universidad Colombiana.

Coraggio, J. L. (2004). Bases para una nueva generación de políticas socioeconómicas: la economía del trabajo o economía popular1. Revista foro, 51, 59-66.

Coraggio, J. L. (2015). La Economía Social y Solidaria (ESS): Niveles y alcances de acción de sus actores. inédito. Recuperado de: www. coraggioeconomía. org.

Castells, M. (2005). La sociedad red. (Vol. 1). Alianza.

Congreso de la República de Colombia. (2009). Ley 1314 de julio 13 de 2009.

Recuperado de: http://www.secretariasenado.gov.co/senado/basedoc/ ley_1314_2009.html

Consejo Técnico de la Contaduría pública (CTCP). (2015) Direccionamiento estratégico Del proceso de convergencia de las normas de contabilidad e información financiera y de aseguramiento de la información, con estándares internacionales. Recuperado de: http://www.ctcp.gov.co/_files/documents/DOC_CTCP_Z6F3T_7.pdf 
Craig, R., Amernic, J., and Tourish, D. (2014). Perverse audit culture and accountability of the modern public university. Financial Accountability \& Management, 30(1), 1-24.

Dacunto, C. y Casinelli, H. (2009). Evaluación de las competencias de los aspirantes a contadores profesionales. Recuperado de: http://www.economicas.uba.ar/wpcontent/uploads/2017/06/SimposioXXXI_M_06_Dacunto_Casinelli.pdf

Fundación IFRS. (2018). Recuperado de: https://www.ifrs.org/

Fundación IFRS. (2017). Marco conceptual NIIF. Recuperado de: http://www.ifrs.org/ Pages/default.aspx2

Gray, R., Adams, C., and Owen, D. (2014). Accountability, social responsibility and sustainability: Accounting for society and the environment. Pearson Higher Ed.

Gray, R., Owen, D., and Adams, C. (1996). Accounting \& accountability: changes and challenges in corporate social and environmental reporting. Prentice Hall.

Huang, G., Baskerville, R., and Fowler, C. (2014). The (Mis) uses of Pierre Bourdieu's practical epistemology in accounting-related social research. (w.i.)

Irigoyen, E. M. (2016). Economía social+ innovación tecnológica: Experiencias de Éxito en Entornos de Precariedad. Journal of technology management \& innovation, $11(1), 86-92$.

Jeacle, I., and Miller, P. (2016). Accounting, culture, and the state. Critical Perspectives on Accounting, 37, 1-4.

Jiménez Escamilla, H. (1997). Contaduría y postmodernidad. (w.i.)

Joseph, M. (2014). Debt to Society: Accounting for Life under. University of Minnesota Press. 
Ledezma, J. (2015). La formación contable en un contexto de normas internacionales. Observatorio de la Universidad Colombiana.

Lux, M., y Pérez, A. (2017). Reflexiones sobre la producción, circulación y uso de las publicaciones académicas en las ciencias sociales. Anuario Colombiano de Historia Social y de la Cultura, 44(1), 125-143.

Mantilla, S. A. (s.f.). Capital intelectual y contabilidad del conocimiento. (s.d.).

Mclntyre, D. D., Chen, C. and Jones, K. T., (2005). A reexamination of the factors important to selection of accounting as a major. Accounting and the Public Interest, $5(1), 14-31$

Presidencia de la República de Colombia. (1993). Decreto 2649 de diciembre 29 de 1993. Recuperado de: https://bibliotecadigital.ccb.org.co/ bitstream/handle/11520/13812/Decreto\%202649\%20de\%201993. pdf?sequence $=1 \&$ isAllowed $=y$

Rhodes, R. A. (1997). Understanding governance: Policy networks, governance, reflexivity and accountability. Open University Press.

Rojas, W., y Ospina, C. M. (2011). Consideraciones sobre el sentido de un proyecto educativo en Contaduría pública. Cuadernos de Administración, 27(45), 45-60.

Tedesco, J. C. (2017). Educación y sociedad del conocimiento y de la información. Revista Colombiana de Educación, (36-37).

Tua Pereda, J. (2012). Contabilidad y desarrollo económico. Contabilidad y negocios, $7(13)$.

Unesco. (1999). Conférence mondiale sur la science, "Projet de Déclaration", UNESCOCIUSS, et "Draft Programme". París. 
Universidad Nacional Abierta y a Distancia, UNAD. (2011). Proyecto Académico Pedagógico Solidario, PAP Solidario. Recuperado de: https://academia.unad.edu. co/images/pap-solidario/PAP\%20solidario\%20v3.pdf

Vessuri, H., Guédon, J. C., and Cetto, A. M. (2014). Excellence or quality? Impact of the current competition regime on science and scientific publishing in Latin America and its implications for development. Current Sociology, 62(5), 647-665.

Walks, A. (2016). A review of Debt to Society: Accounting for Life under Capitalism by Miranda Joseph. (w.i.). 